\title{
MULTINET: Enabler for Next Generation Enterprise Wireless services
}

\author{
Nekane BILBAO ${ }^{1}$, Leire AGINAKO ${ }^{1}$, Oscar LAZARO, Tobias HOF, Christian BONNET, \\ Pablo VAQUERO, Silvia DE LA MAZA, Robert ATKINSON, Benjamin MOLINA, John. \\ O’FLAHERTY, Riccardo MAZZA \\ ${ }^{1}$ Euskaltel, Parque Tecnológico, Edif.809, Zamudio, 48160, Spain \\ Tel: +34 94 4011000, Fax: + 3494 4011297, Email: \\ nbmaron@colaboradores.euskaltel.com,laginako@euskaltel.com
}

\begin{abstract}
Wireless communications are currently experiencing a fast migration toward the beyond third-generation (B3G)/fourth generation (4G) era. This represents a generational change in wireless systems: new capabilities related to mobility and new services support is required and new concepts as individual-centric, user-centric or ambient-aware communications are included. One of the main restrictions associated to wireless technology is mobility management, this feature was not considered in the design phase; for this reason, a complete solution is not already found, although different solutions are proposed and are being proposed. In MULTINET project, features as mobility and multihoming are applied to wireless network to provide the necessary network and application functionality enhancements for seamless data communication mobility considering end-user scenario and preferences. The aim of this paper is to show the benefits of these functionalities from the Service Providers and final User point of view.
\end{abstract}

\section{Introduction}

Enterprises in EU are now focussing their organization and management efforts in the efficient and profitable usage of wireless and mobile technologies. These technologies now offer capabilities for Mobile Office applications (PIM, email, messaging...), that will evolve in the near future into the usage of Mobile Enterprise Applications - ERP, CRM, SCM, PLM. However, to leverage the full potential benefits of wireless communications, it is necessary to improve the underlying network technologies.

Nowadays, considering the final users' change of profile, mobility and new services support is required in any solution and new concepts are proposed as part of $4 \mathrm{G}$ network definition. A number of common needs have been identified as drivers for next generation service enablers. The common needs include:

1. Need for Mobility

2. Need to Accelerate Transmission at users indication.

3. Need to Autonomously Redirect Established Sessions

4. $\quad$ Need to Set Up Preferences

5. Need for Ubiquitous Access

6. Need for Reliability

A solution for seamless data communications through simultaneous networks is developed in order to provide Always Best Connected (ABC) paradigm to the end-user. Mobility, multihoming and network selection capabilities are analyzed to the reference architecture design. 
The paper starts explaining the main objectives of MULTINET project, following the methodology and technology are going to be described in order to use it as based for developments and actually obtained results chapters. Finally associated business benefits and main conclusions will be shown.

\section{Objectives}

The MULTINET communication system must be capable of providing the network and application functionalities transparently to end-user SME companies. In order to provide it, multiple simultaneous networks wants to be seamlessly handled to optimise communications in multiple dimensions; based on new emerging multihoming technology, providing a more intelligent and flexible use of the available wireless infrastructure, and also considering low battery power consumption and better costing. The project aims to provide the necessary networks and application functionality enhancements for seamless data communication mobility.

Nomadic mobility must be provided following request from end-user companies'; High-Tech Machinery Maintenance Companies and Utility Companies' Support Services; mobile worker operation profile. Apart from that the provided services wants to be enhanced, for that, allocation of bandwidth, radio access and network resources is thus managed in an intelligent and coordinated fashion according to the user's state and needs, as depicted in the following picture:

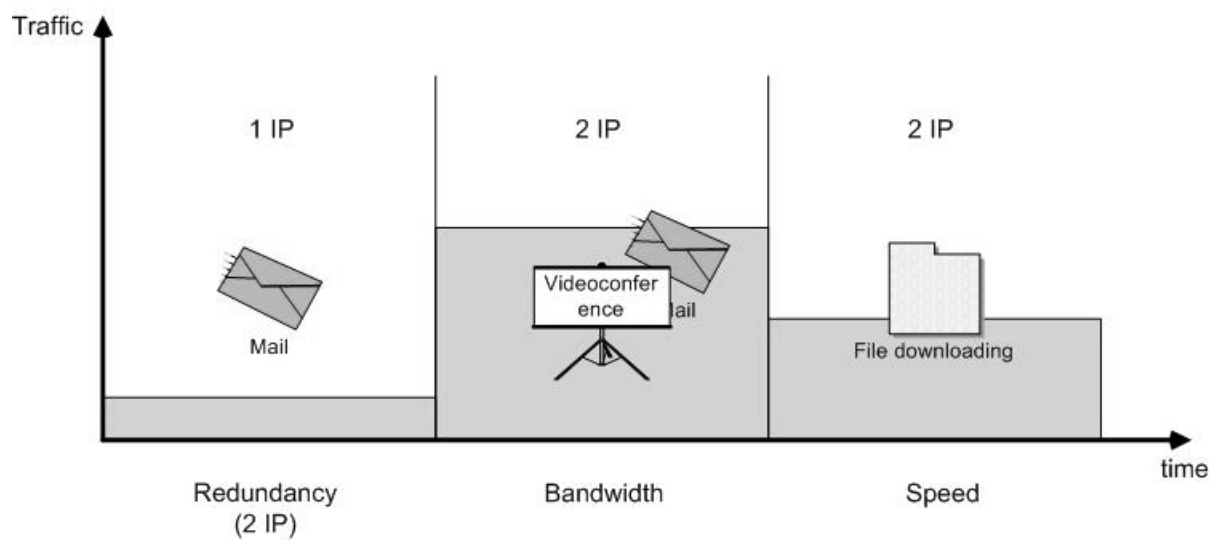

Figure 1-MULTINET intelligent and coordinated resource management

Taking advantage of the various networks interfaces available to the nomadic user in his/her remote location, the availability of multiple IP addresses wants to be exploited by the network so that the user could be provided with increased QoS, higher data rates or respond to dynamic QoS requests made by the user. This use case is related to a situation where a single terminal with a number of air interfaces is available and the single multimodal terminal is multihomed. Mobility could be considered in this case as the situation where a particular application handovers to an alternative point of attachment as a response to QoS variation or load balancing policies.

\section{Methodology}

The project has analysed the requirements from final users (SMEs) and Service Providers, in order to try to collect their main request in terms of access networks, applications used, and necessary QoS. A state of the art analysis related to available solutions for mobility and multihoming management [1] [2] was also performed in order to identify the most suitable solution for the existing scenario and the desired final solution.

Considering the previous points, starting from these technical studies, one of the most important decisions was the selection of the approach for the solution. It was considered the 
project objectives, in particular, seamless perception of the user to network connection, that is, the solution must be transparent to end user but it also must take into account user preferences. In order to provide this, the designed solution is based on a user oriented network centric approach.

Actually, partners are developing technological building blocks in order to satisfy these requirements as much as possible. These blocks will be integrated in following months and trialled with real user SMEs, by the end of year 2007.

\section{Technology Description}

MULTINET is a system supporting mobility and multihoming in order to support advanced service features such as load balancing, enhanced reliability and dynamic bearer allocation. The most suitable network selection is performed to provide it. Following an overview of the used main technologic concepts is included:

\section{1 - Mobility management}

The mobility management is provided using Network Mobility (NEMO) Basic Support protocol [3]. NEMO is an extension of the basic Mobile IPv6 (MIPv6) protocol, this means that the rest of extensions performed in MIPv6 are not supported by default by NEMO. Since NEMO was defined, the progress of two protocols is independent although extensions done in MIPv6 are also considered for provide a complete NEMO solution.

NEMO differs from MIPv6 in its capability to support an entire mobile network opposed to a single mobile node visiting a remote network. The changes introduced by the former respect to the latter relay on the insertion of some new flag and fields in the messages defined in the protocol.

NEMO manages the mobility of a complete Mobile Network (MNet) with one or more Mobile Routers (MR); this MR is a device which acts as the default gateway of a number of devices connected to it, that connects the MNet to Internet to a fixed or wireless backbone. It can change its point of attachment to other networks as it physically moves or changes in topology. The mobility is transparent for the nodes that compose the MNet, and allows session continuity for every node within it as the network moves. The communication that RFC 3965 defines is done using a bi-directional tunnel between MR and Home Agent (HA), the next image summarizes the communication process:

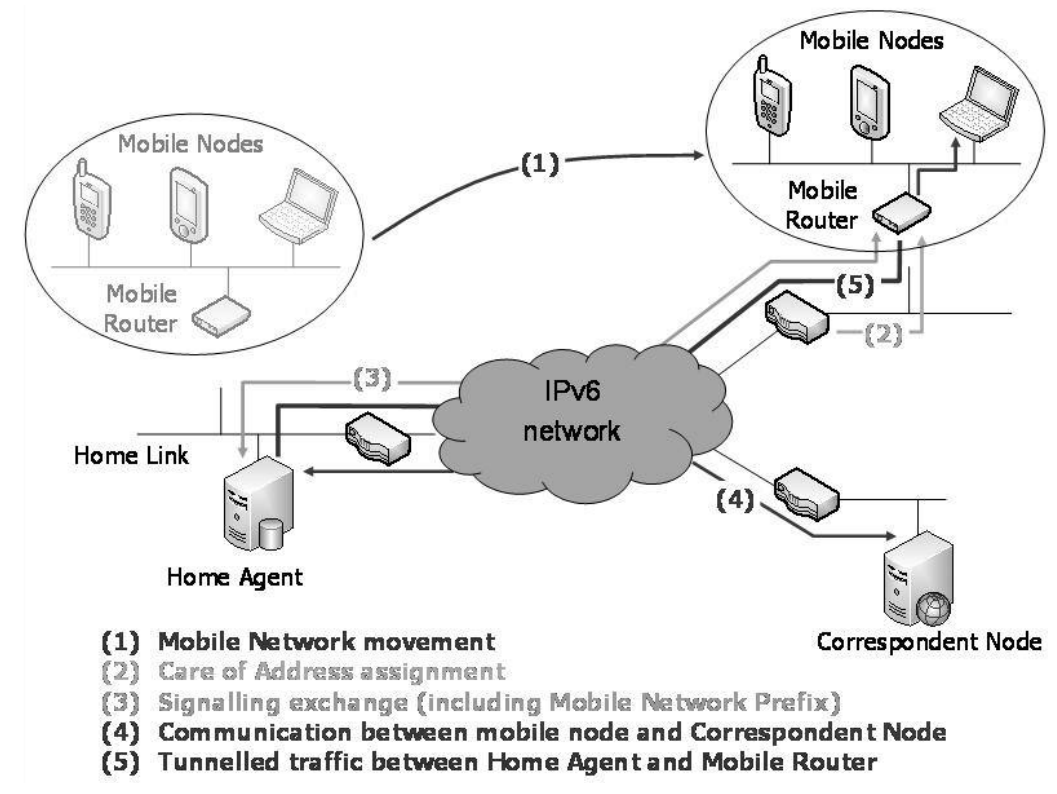

Figure 2 - NEMO architecture 
This architecture is suitable for Personnel Area Networks (PANs), but is extensible to any scenario in which the network and its mobility want to be managed as a whole structure; thanks to this approach, user terminals do not require mobility support and are not aware of mobility management issues.

\section{2 - Multihoming management}

The multihoming is the ability to have multiple network addresses for a unique terminal to identify its multiples and simultaneous connections to the network; this multiple addresses can be associated to a single or multiple interfaces. Multihoming solutions can offer a wide spectrum of services depending of requirements of the designed architecture and desired solution.

The multiple points of attachment are used to provide mainly two types of service, load balancing and redundancy. The first one implies parallel simultaneous streams but the second only specifies the desired stream between the existing possibilities in each case, the parallel concept is not covered. The normal scenario consists in providing a remote network multiple paths to connect to the Internet, as depicted in the next figure:

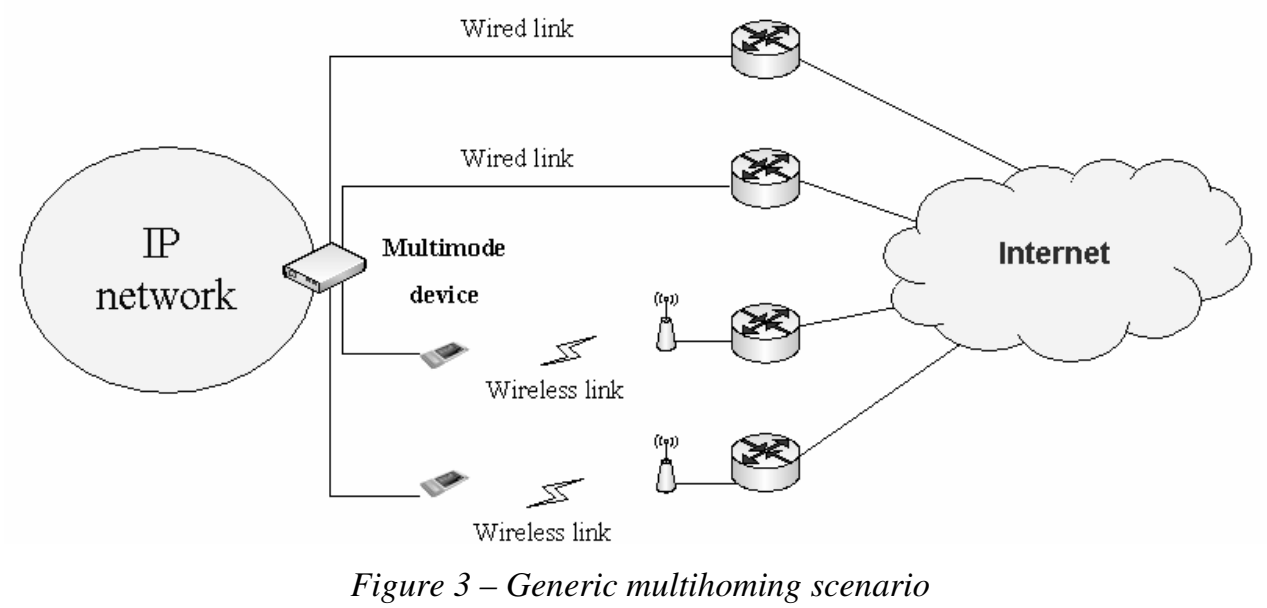

It is relevant to note that the common goal of the multihoming and mobility solutions is to provide a communicating node with uninterrupted connectivity throughout changes in the attachment point. In the mobility scenario, the communicating end-point, changes its attachment point because of its movement. In the MULTINET nomadic multihoming scenario, the attachment point used by the endpoint for communication varies because of topological changes caused by outages in the communication elements. While the causes are different, there seems to be enough similarities between the two scenarios to consider common solutions. The multihoming scenario can be mapped into a mobility scenario.

In the solution for this project, the mobility abstraction of the node, allows a more centralized management that can be also used for multihoming centralization. NEMO protocol definition does not discuss multihoming for Mobile Routers but Multiple Care of Address (MCoA) proposals for MIPv6 can be extrapolated to the NEMO scenario.

According to MIPv6 specification, a mobile node cannot register multiple Care-Of Addresses for a single Home Address: If a Mobile Node sends Binding Updates for different Care-Of Address the Home Agent always overwrites the Care-Of Address recorded in the binding cache with the one contained in the last received Binding Update. The Multiple Care-of Addresses draft [4] proposes a MIPv6 and NEMO extension that allows a Mobile Node or a Mobile Router to register multiple Care-Of Addresses to a single Home Address.

The MCoA extension introduces an identification number called Binding Unique Identifier (BID) to distinguish the Bindings Updates from the same Mobile Node and 
corresponding to his different interfaces. So the Home Address identifies a Mobile Node itself and the BID identifies the bindings for a given interface. The BID can then also be used as a search key for a corresponding entry in the binding cache in addition to the Home Address. In order to register multiple Care-Of Addresses, the Mobile Node sends a binding update containing a Binding Unique Identifier sub-option. This is an extension of the standard BU message, which contains the BID corresponding to the Care-Of address to be registered.

In this system; NEMO with MCoA extension; Nautilus flow distribution schema [5] is applied to manage existing different flow and provide a suitable multihoming solution.

\section{3 - Network Selection Algorithm (NSA)}

The aim of the system is to ensure ABC paradigm, for this objective a dynamic selection of the most suitable access network must be developed between simultaneously available ones. The selection depends on wireless link measurements based on RSSI and SNR parameters, network load status and user preferences. The decision taken by the algorithm activates the handover process and reassign the flow to the best interface.

NSA represents the intelligence in the MULTINET system and is related to the selforganisational aspect of the network management strategy devised as the enabler for next generation service. In the context of MULTINET, NSA is the mechanism selected to determine how the multihoming strategy could be best exploited. This means that NSA makes decisions in terms of assigning flows to particular interfaces. It is located on the core of the system and interacts with all the functional blocks included in the system.

\section{Developments}

One of the main tasks of the development was the design of the reference architecture. There are several groups working to obtain a common framework as solution for scenarios including heterogeneous networks but there is not still a mature solution. The architecture design takes into account the running proposals and includes scenario specifications and conclusions from the previous work and it is used as guidance during all the development process. In the next figure the designed reference architecture is shown:

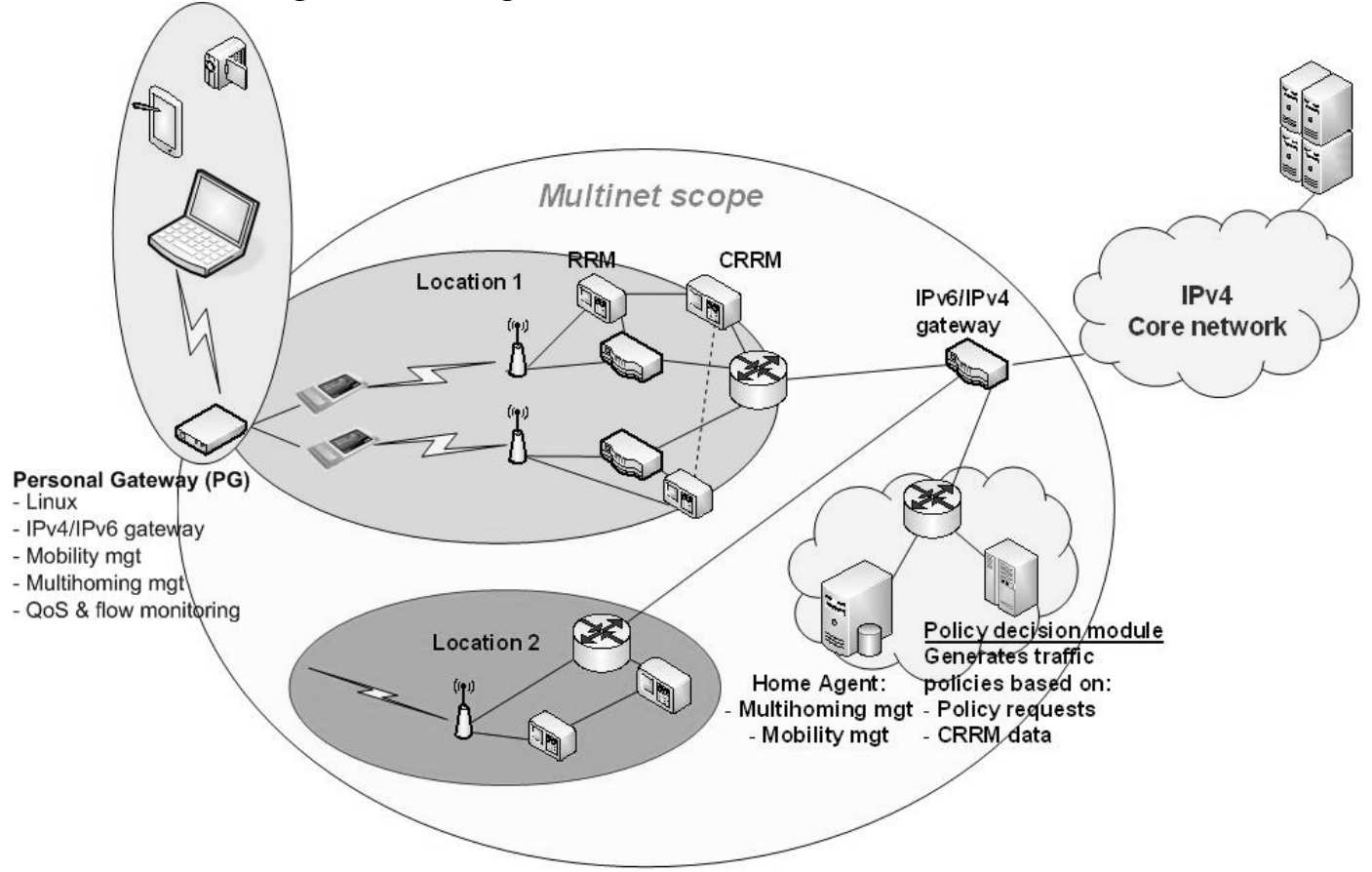

Figure 4 - Reference architecture 
The main modules and their functionalities are described below:

1. Personal Gateway (PG): It has got the role of the Mobile Router of NEMO architecture but also includes other functionalities associated to network selection and multihoming. One of the functionalities is the MTC that measures the link quality of the access networks detected by the PG. It measures the SNR and RSSI values that will be used for network selection. In the other side and considering that it is the unique device connected to the end-user it is the responsible of redirecting the user preferences also to the network selection.

2. CRRM-RRM-Wimeter: This schema is based on the Wimeter sniffing tool that is connected to each Access Point. It measured the available bandwidth and load in the network that will be used as an input for the network selection algorithm.

3. Policy decision module is responsible of network selection; it receives the different inputs from the system and generates the triggering to activate the handover in the HA.

4. IPv6/IPv4 gateway: The user applications and are design for IPv4 support and must be maintained unchangeable but an efficient mobility and multihoming solution must be performed in IPv6.

The modules are independently developed and tested and the total system Data Model and Operation Flow are being discussed in order to start with the integration phase.

\section{Results}

The project is still running so the results of the overall solution will not be available until end of the year. Even so, some of the functional blocks from the MULTINET architecture have been already tested.

\section{1 - Radio resource monitoring}

The traffic load is measured by capturing all frames sent over the air interface using wimeter sniffing tool and it is able also to differentiate traffic according to their type (control, data, unicast, broadcast), the transport protocol, the receiver/sender, the application, etc.In the next figure the estimation of the variation of the available bandwidth is shown:

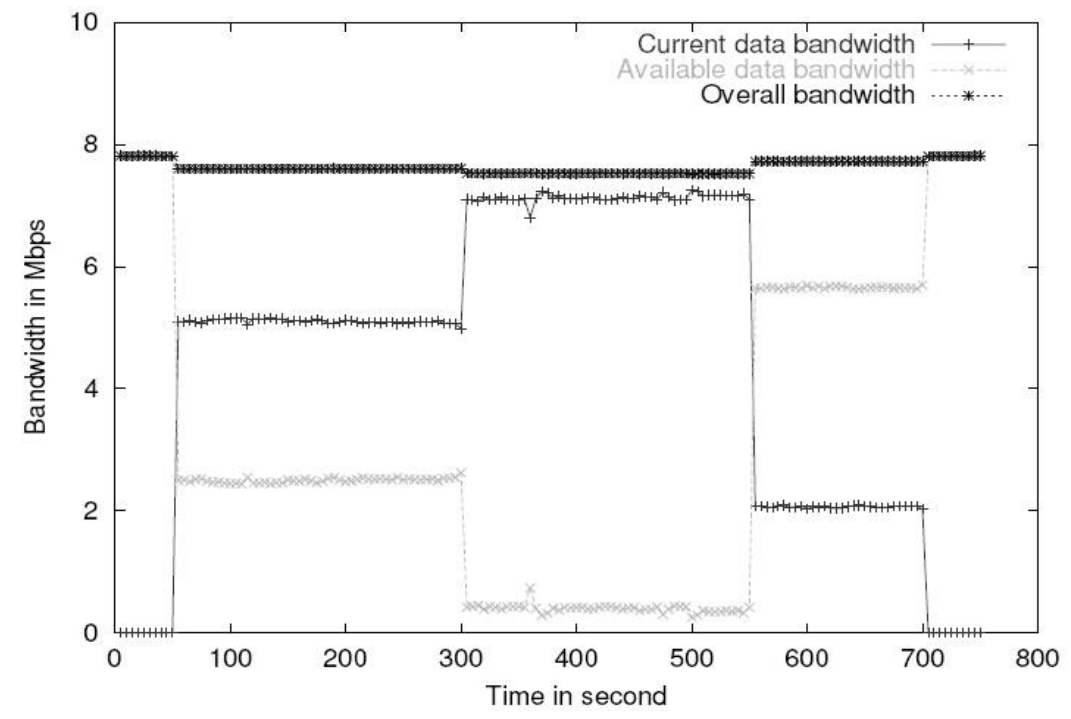

Figure 5 - Wimeter bandwidth measurement 


\section{2 - User preferences}

The user preferences are not going to directly trigger the handover process, although they are going to be considered in the network selection. This is due to the system follows a user oriented network centric approach. The user preferences profile is already defined contains terminal capabilities, information associated to the user itself, user desired Service Level or Quality of Service and referring to access network characteristics such as cost.

\section{3 - Mobility and multihoming management}

An architecture that provides mobility and multihoming management is already developed and tested. This architecture includes NEMO mobility protocol with MCoA registration extension applied to provide multihoming capability. A Policy Reception and Enforcement Module is also included in order to simulate the handover generated by Network Selection Algorithm. The next figure reproduces the testbed:

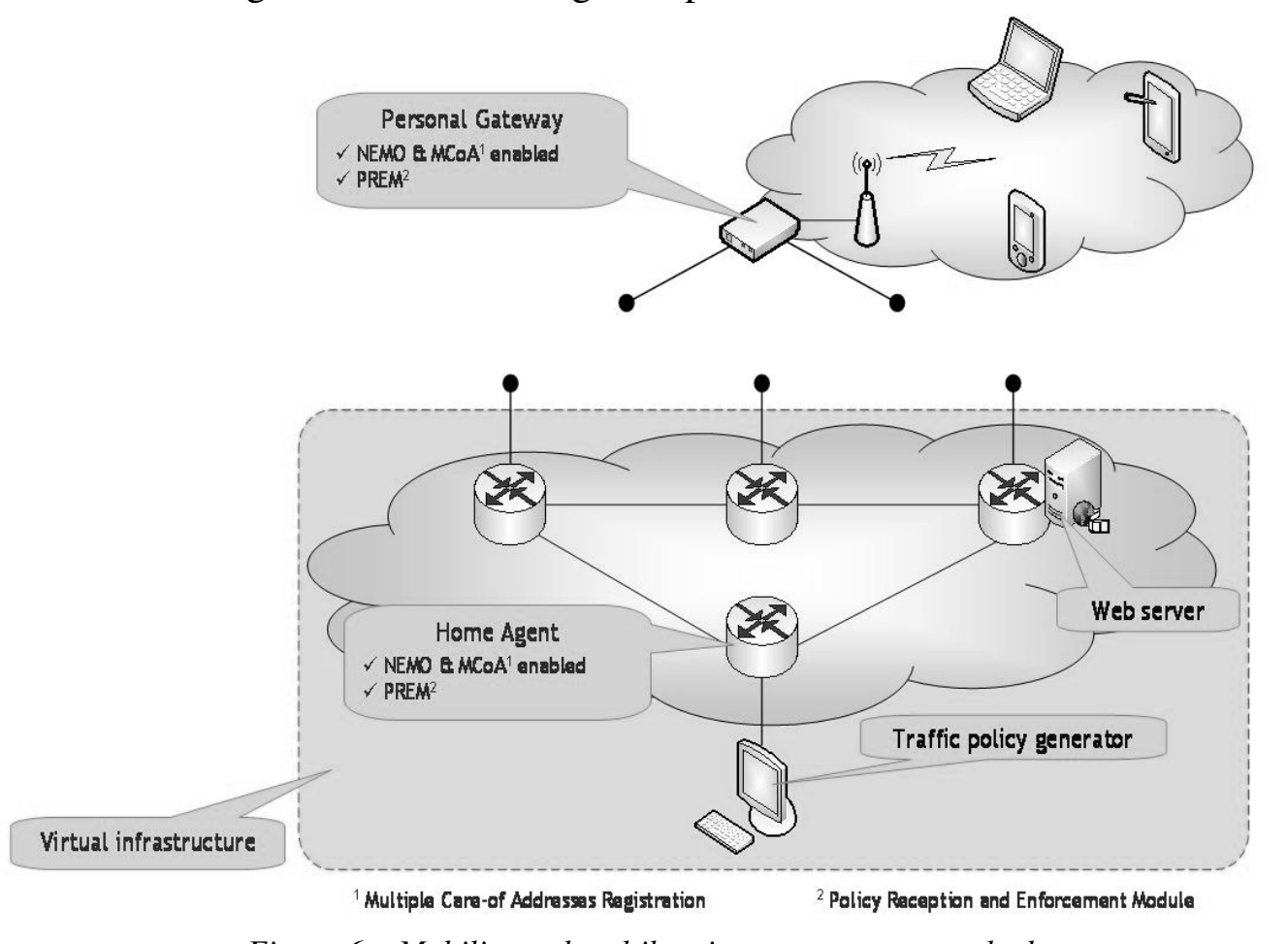

Figure 6-Mobility and multihoming management testbed

As result, it is checked that the seamless connectivity between simultaneous networks is offered. VLC video application (this application does not include got any buffer) was used to demonstrate that traffic was correctly redirected between available networks without any disruption in the traffic.

\section{Business Benefits}

This solution implies business benefits not only in Telecommunication Operator or Service Providers, but also for potential user companies. Following the main benefits are listed:

1. The MULTINET technologies are oriented but not limited to the nomadic working force. The services enabled include user driven fast data transfer and adaptive video streaming sessions.

2. Final user, enterprises and SMEs can obtain benefits through the new multimedia services that permit managing their activities from anywhere, with reduced battery power consumption, terminal capabilities and better pricing conditions. Cost reduction in terms 
of mobile worker operation time reduction and the cost of the selected network for transmission.

3. Ad-hoc network use, through selection of the most suitable communication network. The MULTINET system will be provided with an intelligent mechanism to select the most suitable communication network, depending on the type of data to be transmitted, the network radio parameters and user preferences.

4. Increase personnel efficiency, by the reduction of the response time in delivering the service and increase of service accuracy which will lead to more tasks successfully accomplished on time.

5. The designed solution is transparent to the end-user, it must be taken into account that they do not need to be ICT technical users and the supported applications must remain unchangeable.

6. Reduction of support services answer time, increasing customer satisfaction.

7. An increased services portfolio will be offered, the increased capabilities of the mobile field force and the communication technology used, will allow the development of new services based on the access to multimedia data.

As a consequence of these benefits, MULTINET will provide all of its users with an increased competitiveness in relation to their competitors. It is also expected that vendors will incorporate such a technological advance in their turnkey solution portfolio, for the provision of integrated digital telecommunication systems. In the case of Service Providers or Telecommunication Operators, a better use of the available resources will be performed. The resources will not be locked to users independently of their requirements, it will be assignment more optimum depending of their needs and requirements. This will imply also a cost benefit for the end user point of view.

\section{Conclusions}

In this project simultaneous WLAN access networks are used to provide a mobility and multihoming enhanced solution. This solution is combined with the most suitable access network selection following $\mathrm{ABC}$ paradigm to provide the explained objectives. Network radio status and user preferences are considered for the interface selection. A NEMO based architecture is being develop, applying MCoA extension to attach multihoming capabilities to it. This architecture generates a handover decision and triggering generated by Network Selection Algorithm that provides benefits to the established and new sessions.

As further work it is considered for the introduction of different access technologies; WiMAX and UMTS. This way the pilot will support not only WLAN-WLAN scenarios, it will be applicable for WLAN-WiMAX and WLAN-UMTS. The Consortium considers this technology can provide business users with new tools to improve productivity and reduce costs.

\section{References}

[1] MULTINET IST-27437 STP D2.1 v1.0-RE.pdf

[2] MULTINET IST-27437 STP D2.2 v1.0-RE.pdf

[3] Devarapalli V., Wakikawa, R., Petrescu, A., and P. Thubert, "Network Mobility (NEMO) Basic Support Protocol", RFC 3963, January 2005

[4] Wakikawa, R., Ernst, T. and Nagami, K., "Multiple Care-of Addresses Registration", draft-ietf-monami6multiplecoa-01.txt (work in progress), June 2006

[5] Mitsuya, K., Tasak, K, and Wakikawa, R., "A Schema Fragment for Flow Distribution”, draft-mitsuyamonami6-flow-distribution-policy-02.txt (work in progress), June 2006.

[6] Nautilus6: http://www.nautilus6.org/ : MCoA implementation

[7] Soliman, H., "Flow Bindings in Mobile IPv6", draft-soliman-monami6-flow-binding-02.txt (work in progress), September 2006 